\title{
Habilidades sociales: desarrollo desde lo lúdico, en niños de etapa pre escolar
}

\author{
María Patricia Unuzungo Preciado \\ p7002323567@ucvvirtual.edu.pe \\ https://orcid.org/0000-0001-2167-2530 \\ Universidad "César Vallejo" Piura - Perú \\ César Balladares Atoche \\ cballadaresa@ucvvirtual.edu.pe \\ https://orcid.org/0000-0001-8242-7742 \\ Universidad "César Vallejo" Piura - Perú \\ Betsy Janeth Bravo Cedeño \\ p7002325469@ucvvirtual.edu.pe \\ https://orcid.org/0000-0002-5875-7968 \\ Universidad "César Vallejo" Piura - Perú \\ Cristina Verónica Gordon Torres \\ p700232573@ucvvirtual.edu.pe \\ https://orcid.org/0000-0001-5192-2097 \\ Universidad “César Vallejo” Piura - Perú \\ Ludia Marisol Quito Santana \\ p7002325162@ucvvirtual.edu.pe \\ https://orcid.org/0000-0003-4103-6467 \\ Universidad "César Vallejo" Piura - Perú \\ Giovanni David Fernandez Unuzungo \\ p7002489954@ucvvirtual.edu.pe \\ https://orcid.org/0000-0002-2986-2865 \\ Universidad “César Vallejo” Lima - Perú
}

\section{RESUMEN}

La investigación tuvo como finalidad proponer las Habilidades Sociales en estudiantes del centro de educación inicial Los Rosales, Amie 23H00092 Santo Domingo El diseño que se aplicó es no experimental, descriptivo, propositivo de corte transversal, que de acuerdo a las intencionalidades del recojo de información se optó por la guía de observación, como instrumento la lista de cotejo, la muestra estuvo integrada por 50 estudiantes con edades promedio de tres años, en los estudiantes, se elaboró un instrumento para recoger información sobre las habilidades sociales el mismo que fue 
validado por expertos, se cumplieron con las exigencias de la confiabilidad para estar apto en su aplicación, en este sentido se utilizó el coeficiente Alpha de Cronbach. En este sentido el coeficiente de fiabilidad fue de 0.89. De acuerdo a los resultados comparados entre el pretest y postest, la conclusión fue que la aplicación de las estrategias lúdicas mejora significativamente el desarrollo psicomotor de los niños objetos de investigación. El conjunto de estrategias lúdicas, para aplicarlas se consideró la edad del niño, el aporte teórico de investigadores que han estudiado el desarrollo evolutivo del niño, del orden y coherencia de las actividades de acuerdo a la complejidad del tema.

Palabras clave: habilidades sociales; programa lúdico; estudiantes; desarrollo psicomotor 


\title{
Social skills: development from play, in pre-scholl children
}

\begin{abstract}
The purpose of the research was to propose Social Skills in students from the Los Rosales initial education center, Amie 23H00092 Santo Domingo The design that was applied is non-experimental, descriptive, propositional of cross-section, which according to the intentions of the information collection is opted for the observation guide, as an instrument the checklist, the sample was made up of 50 students with an average age of three years, in the students, an instrument was developed to collect information on social skills, which was validated by experts, the reliability requirements were met to be suitable in its application, in this sense the Cronbach's Alpha coefficient was used. In this sense, the reliability coefficient was 0.89 . According to the results compared between the pretest and posttest, the conclusion was that the application of playful strategies significantly improves the psychomotor development of the children under investigation. The set of playful strategies, to apply them, the age of the child was considered, the theoretical contribution of researchers who have studied the evolutionary development oh the child, the order and coherence of the activities according to the complexity of the subject.
\end{abstract}

Keywords: social skills, Playful program, students, psychomotor development

Artículo recibido: 30 noviembre. 2021 Aceptado para publicación: 29 diciembre 2021 Correspondencia: p7002323567@ucvvirtual.edu.pe Conflictos de Interés: Ninguna que declarar 


\section{INTRODUCCIÓN}

La infancia, es fundamental porque allí es donde se establecen las primeras interacciones de apego que ayudan al desarrollo emocional de los individuos. Por medio de estos primeros vínculos, se construye su identidad, seguridad, autoestima y autonomía (García, 2021). Esta descripción se evidencia cuando los individuos van desenvolviéndose, de acorde a sus posibilidades, les permite construir su identidad, originando que se den cuenta de sus gustos, preferencias, necesidades e intereses. Por otro lado, UNICEF (2021) considera que "Sin emociones no hay colorido; es la emoción la que da un sello a lo que realizan a cada paso" es justamente estos pasos que se van marcando en las experiencias a través de las habilidades sociales que se van desarrollando y consolidando a través de los cuidados y sobre todo de las personas que rodean a los niños.

Por lo anteriormente expuesto, nos permite tener en cuenta que para poder socializar se debe establecer las habilidades sociales tal como lo señala UNICEF (2021a) para el caso de Argentina encontró que un $38.56 \%$ de los estudiantes presentan un nivel en proceso de desarrollo de sus habilidades sociales, en donde se han establecido acciones como el interactuar, involucra habilidades comunicativas, colaborativas, resolución de problemas y negociación para asumir compromisos.

Por otro lado, de acuerdo al acápite anterior tenemos la UNESCO (2020) indica que las habilidades sociales y emocionales representa el gran reto de la educación del futuro; es por ello que ha fundamentado que es imprescindible, el desarrollo de habilidades en el aprendizaje del siglo XXI; habilidades comunicativas, colaborativas destacando la importancia del conjunto de competencia personal y social (capacidades que desarrollen en la persona la iniciativa, resiliencia, responsabilidad, creatividad, la cooperatividad, compromiso con respecto al trabajo en equipo, en red, empatía, solidaridad y compasión). Todas las habilidades mencionadas anteriormente son indispensables, para acceder al campo laboral, como para convivir en el contexto social.

Rentería (2018) investigó sobre "Habilidades sociales y convivencia escolar en Ferreñafe"- Perú. El tipo de investigación fue propositiva enmarcada en el el enfoque cuantitativo, como diseño se consideró el no experimental, descriptivo, transversal propositivo. Para obtener los datos de las variables se utilizó los cuestionarios para una muestra no probabilística conformada por 167 estudiantes. Como resultado tenemos que las habilidades sociales de los docentes es de $89 \%$ esto significa que existen un ben nivel 
de habilidades sociales que se manifiestan en buenas relaciones entre los estudiantes y docentes, sin embargo el $11 \%$ restante presentan actitudes poco adecuadas en la institución educativa, las mismas que se ponen de manifiesto en no respetar las ideas de sus compañeros, y cuando se presentan dificultades no buscan alternativas de solución dejando las la responsabilidad a otros miembros de la institución educativa.

Araujo (2017), el autor desarrollo la tesis titulada "Habilidades sociales y desempeño docente". De acuerdo a la revisión bibliográfica el tipo de investigación fue correlacional, dentro del enfoque cuantitativo; el diseño de investigación es el no experimental descriptivo correlacional de corte trasversal. La muestra se conformó por 60 docentes, con un muestreo no probabilístico a juicio del investigador, Para el recojo de los datos se utilizó la técnica de la encuesta con su instrumento: los cuestionarios sobre: habilidades sociales y desempeño docente. Como resultado tenemos que las habilidades sociales, se observó que el 75\% de participantes de la investigación indicaron que las habilidades sociales son buenas, sin embargo, un $25 \%$ considera que es regular. Teniendo en cuenta estos resultados sobre todo a la variable en habilidades sociales se puede concluir que la falta mejorar las relaciones sociales, el buen trato y respeto entre docentes, esto debido a que existen diferencias en la conducción de la entidad educativa.

En relación a la variable programa lúdico se considera como una elección de comprender el contexto, concibiéndose una nueva representación que transforma de forma creativa la manera de percibir los hechos, fenómenos, acontecimientos que ocurren en diferentes contextos, originándose el nuevo procedimiento cognitivo, creativo y de interrelación emocional positiva (Dinello 2007, p. 22)

Por otro lado, Rojas (2001) considera al programa lúdico como el conjunto formado por estrategias que tienen la finalidad de generar condiciones de espacio estético en el cual los niños construyan, vivencien su aprendizaje. Este programa tiene una metodología cuya finalidad es buscar que los niños aprehendan los contenidos que los docentes imparten a través de la estrategia lúdica. La metodología lúdica no implica únicamente jugar para recrearse, sino por el contrario logra desarrollar experiencias intensas en las cuales el niño aprehende naturalmente. Rojas, fundamenta que durante los tres primeros años de edad se debe realizar el juego sensorial, con el transcurrir el tiempo se recomienda realizar juegos que promuevan el proceso de imaginar y finalmente es recomendable promover juegos de competencia. 
En México, se brinda mucha importancia a las habilidades sociales es por ello que a través del Facebook se desarrollan programas "Escuela para Padres online" en donde su propósito radica en dar a conocer que la falta de habilidades sociales genera conductas disfuncionales en los niños. En este sentido Abrams, et al. (2016) indica que la voz de la madre es muy importante porque se convierte en un elemento emocional y social en su proceso de desarrollo; el mismo que impulsa las destrezas expresivas y sociales de los niños; el último de vital importancia para el desarrollo de las habilidades sociales en los niños a temprana edad.

En la República del Ecuador, de acuerdo a las exigencias de desarrollar habilidades sociales existen instituciones educativas como es el caso de la Unidad Educativa Thomas More, que aplica metodologías de enseñanza de varios países (Finlandesa, Singapur, Alemana y Estados Unidos) adaptada al sistema educativo ecuatoriano, llamada 4C's Learning System que trabajan en 4 competencias claves para que los alumnos se desenvuelvan en la sociedad como: Creatividad, Criticidad, Comunicación y Cooperación (Martínez, 2021).

En relación a la justificación la presente investigación se sustentó teóricamente en comprender los aportes teóricos sobre las habilidades sociales, los mismos que fundamentan el aspecto interpersonal de los niños, además de comprender las teorías del juego desde la posición de varios autores, considerando que es una actividad lúdica que se ajusta a las expectativas e inquietudes de los niños. Estas teorías son fundamentales para la estructuración de la propuesta que buscó consolidar el desarrollo activo y emocional de los niños en donde se pone de manifiesto sus estados emocionales de sus compañeros. Es oportuno indicar que con el conocimiento de las teorías se conoce el nivel de evolución de las actividades y sobre todo el desarrollo cognitivo del mismo.

Además, tenemos la relevancia práctica, en donde es primordial el conocimiento de las características de los niños en relación a las habilidades sociales, es importante conocer los estados comunicacionales, la interacción, sus emociones y sobre todo las relaciones interpersonales, razón primordial que los profesionales de la educación inicial les permita desarrollar estrategias y actividades acorde al nivel de los niños, con la finalidad de ejercer una didáctica específica en la formación de los mismos.

El juego como actividad es un elemento fundamental para desarrollar el proceso de socialización, pues permite al ser humano y en este caso específico al niño aflorar sus 
cualidades y habilidades para comprender, analizar, y sobre todo tener en cuenta criterios de convivencia en cual se desarrollen actitudes de forma pacífica y armoniosa con la finalidad de desarrollar una cultura de paz (Bruner, 1984, p. 250).

Por otro lado, se presenta la justificación metodología en donde se elaboró un instrumento para recoger información sobre las habilidades sociales el mismo que fue validado por expertos y se cumplieron con las exigencias de la confiabilidad para estar apto en su aplicación. Por último, la relevancia social nos permitió comprender la importancia que significa conocer las habilidades sociales, toda vez que se diagnosticó y comprendió los diversos comportamientos de los niños, y a partir de allí solucionar los problemas interpersonales, razón fundamental para que los niños se involucren en el crecimiento armónico y solidario entre compañeros.

La dimensión desarrollo de sentimientos y emociones según Roca (2014) nos permite identificar y reconocer los diversos elementos que se presentan en el accionar de las personas, y que muchas veces pasan desapercibidos en nuestro inconsciente. Esta dimensión autorregula el comportamiento de las personas el mismo que se cumple al poner en práctica una serie de valores y condiciones que se deben cumplir para justamente controlar el estado emocional de los demás.

\section{ESTRATEGIAS METODOLÓGICAS O MATERIALES Y MÉTODOS}

La investigación es de tipo básica, debido a la finalidad que comprende el proceso investigativo, según Lakatos (1998) "es el proceso que consiste en recoger información de un fenómeno determinado con la finalidad de ahondar en el conocimiento de la realidad estudiada". El diseño que se aplicó es el no experimental, descriptivo, propositivo de corte transversal, esto se manifiesta en la no manipulación de variables, por otro lado, es preciso manifestar se administró en un solo tiempo los instrumentos para el recojo de los datos por lo tanto se considera de corte transversal. Después del diagnóstico se planteó la propuesta que busca convertirse en un medio fundamental para solucionar los problemas identificados en la variable diagnosticada. (Carrasco, 2009). La población estuvo conformada por 50 estudiantes, 24 mujeres y 26 varones. Para la muestra de acuerdo con Valderrama (2013) que es "un subconjunto de la población que está siendo estudiada", en este sentido de acuerdo a la investigación se consideró la totalidad de la población, es decir 50 estudiantes de la institución educativa. 


\section{RESULTADOS Y DISCUSIÓN}

Los resultados están en función al objetivo específico 1: Diagnosticar el nivel de habilidades sociales en estudiantes de etapa pre escolar

\section{Tabla 1}

Variable habilidades sociales

\begin{tabular}{ccc}
\hline Nivel & Frecuencia & Porcentaje \\
\hline Alto & 2 & $4 \%$ \\
Medio & 48 & $96 \%$ \\
Bajo & 0 & $0 \%$ \\
Total & $\mathbf{5 0}$ & $\mathbf{1 0 0 \%}$ \\
\hline
\end{tabular}

Nota. En la Tabla 1 se puede evidenciar que un $96 \%$ de los participantes que respondieron sostienen que las habilidades sociales se encuentran en el nivel medio, asimismo un mínimo $4.0 \%$ considera que se encuentra en el nivel alto. Estos resultados nos indican que las dimensiones de la variable en mención deben replantearse para que mejoren los resultados en relación al comportamiento y actitudes de los niños.

\section{Tabla 2}

Dimensión comunicación afectiva de las habilidades sociales

\begin{tabular}{ccc}
\hline Nivel & Frecuencia & Porcentaje \\
\hline Alto & 1 & $2 \%$ \\
Medio & 37 & $74 \%$ \\
Bajo & 12 & $24 \%$ \\
Total & $\mathbf{5 0}$ & $\mathbf{1 0 0 \%}$
\end{tabular}

Nota. En la Tabla 2 se puede evidenciar que un $74 \%$ de los participantes que respondieron sostienen que las habilidades sociales se encuentran en el nivel medio, además de un mínimo $2 \%$ considera que se encuentra en el nivel alto., por otro lado, tenemos un $24 \%$ de los estudiantes indican que las habilidades sociales están en un nivel bajo. Estos resultados nos indican que las dimensiones de la variable en mención deben replantearse para que mejoren los resultados en relación a promover técnicas dialógicas, y ausencia de habilidades comunicativas, en consecuencia, se necesita diseñar la propuesta.

\section{Tabla 3}

Dimensión interacción social de las habilidades sociales

\begin{tabular}{ccc}
\hline Nivel & Frecuencia & Porcentaje \\
\hline Alto & 1 & $2 \%$ \\
Medio & 43 & $86 \%$ \\
Bajo & 6 & $12 \%$ \\
Total & $\mathbf{5 0}$ & $\mathbf{1 0 0 \%}$ \\
\hline
\end{tabular}


Nota. En la Tabla 3 los resultados obtenidos se evidencian que el 86\% considera que la dimensión interacción social se presenta en un nivel medio, además de un $2.0 \%$ que considera que se ubica en el nivel alto; asimismo tenemos que el $12 \%$ de la muestra indica que la interacción social se establece en el nivel bajo. Estos resultados nos indican que se presentan dificultades para intercambiar ideas respetando los momentos, esto significa que existe discrepancia y falta de amabilidad y cortesía en el acto comunicativo, por otro lado, tenemos que existe confianza entre los niños que creen historias y las socialicen con sus compañeros demostrando empatía y cooperación.

\section{Tabla 4}

Dimensión Desarrollo de sentimientos y emoción de las habilidades sociales

\begin{tabular}{ccc}
\hline Nivel & Frecuencia & Porcentaje \\
\hline Alto & 2 & $4 \%$ \\
Medio & 45 & $90 \%$ \\
Bajo & 3 & $6 \%$ \\
Total & $\mathbf{5 0}$ & $\mathbf{1 0 0 \%}$ \\
\hline
\end{tabular}

Nota. En la Tabla 4 se puede evidenciar que un $90 \%$ de los participantes que respondieron consideran que la dimensión desarrollo de sentimientos y emociones se encuentra en el nivel medio, además de un $4.0 \%$ que considera que es encuentra en el nivel alto, sin embargo, encontramos un $6 \%$ en el nivel bajo. Estos resultados nos indican que se autorregula el comportamiento y sobre todo tiene un control de sus emociones, en consecuencia, manifiesta sus inquietudes de forma espontánea sin ocultar sus sentimientos, por otro lado, los resultados muestran que el niño opina sin incomodar o agredir a su compañero, resaltando su amistad y empatía frente a los demás; es oportuno indicar que algunas de estas acciones no se cumplen.

\section{Tabla 5}

Dimensión resolución de conflictos de las habilidades sociales

\begin{tabular}{ccc}
\hline Nivel & Frecuencia & Porcentaje \\
\hline Alto & 3 & $6 \%$ \\
Medio & 47 & $94 \%$ \\
Bajo & 0 & $0 \%$ \\
Total & $\mathbf{5 0}$ & $\mathbf{1 0 0 \%}$ \\
\hline
\end{tabular}

Nota. En la Tabla 5 se puede evidenciar que un $94 \%$ de los participantes que respondieron consideran que la dimensión resolución de conflictos se encuentra en el nivel medio, además de un $6.0 \%$ que considera que se ubica en el nivel alto. Estos resultados nos 
indican que los niños pueden manifestar sus inquietudes y preocupaciones sin llegar agredir a sus compañeros, es más cuando siente que incomoda pide las disculpas necesarias y sobre todo busca soluciones sin agredir a nadie, planteando acuerdos para la solución de problemas.

\section{Tabla 6}

Dimensión relaciones interpersonales de las habilidades sociales

\begin{tabular}{ccc}
\hline Nivel & Frecuencia & Porcentaje \\
\hline Alto & 2 & $4 \%$ \\
Medio & 46 & $92 \%$ \\
Bajo & 2 & $4 \%$ \\
Total & $\mathbf{5 0}$ & $\mathbf{1 0 0 \%}$ \\
\hline
\end{tabular}

Nota. En la Tabla 6 se puede evidenciar que un $92 \%$ de los participantes que respondieron consideran que la dimensión relaciones interpersonales se encuentra en el nivel medio, además del $2 \%$ se considera en el nivel alto, asimismo tenemos que un $2 \%$ se encuentra en el nivel bajo. Estos resultados nos indican que se acomoda a las necesidades de sus compañeros con la intención de generar amigos, demostrando empatía ente todos, asimismo con un afán de integrarse a los grupos crean composiciones narrativas con lenguaje claro y sencillo.

En relación a la variable habilidades sociales tenemos el aporte teórico Escales, Pujantell (2016) el cual establece que la teoría sociocultural se convierte en una parte fundamental de las habilidades de la persona que se originan en el contexto social.

La dimensión comunicación afectiva con el aporte teórico de Roca (2014) manifiesta que es un proceso entre dos o más personas permitiéndole adquirir conocimiento, formar relaciones sociales, solucionar conflictos, establecer acuerdos; asimismo tenemos la autoestima que implica la autoevaluación o juicio que las personas hacen de sí mismo, sea positivo o negativo.

Para la dimensión interacción social tenemos el aporte de Olivos (2010) el cual manifiesta que debe "existe buenas relaciones entre los seres humanos, demostrando amabilidad y cortesía que son muestras esenciales para el buen trato de las personas", estos elementos deben ser desarrollados por los niños, el mismo que les permita interactuar de forma empática y a temprana edad en diversos contextos sociales, el mismo que les permite adquirir un conjunto de habilidades y destrezas para poder comunicar y expresar sus pensamientos y emociones frente a los demás. 
En relación a la dimensión desarrollo de sentimientos y emociones según el aporte de Roca (2014) permite identificar y reconocer los diversos elementos que se presentan en el accionar de las personas, y que muchas veces pasan desapercibidos en nuestro inconsciente.

Para la dimensión resolución de conflictos tenemos el aporte de Barrera, et al. (2017) sostiene que se debe aplicar estrategias y procedimiento en el cual se soluciones los problemas de forma armoniosa donde sobresalga el diálogo el cual debe ser parte fundamental en la formación de los niños,

Para la dimensión relaciones interpersonales tenemos el aporte de Olivos (2010) en donde concibe que es fundamental reconocer que juegan un factor primordial en el desarrollo integral del ser humano, en ese sentido favorece su adaptación integración en diferentes contextos.

\section{CONCLUSIONES O CONSIDERACIONES FINALES}

De acuerdo a los resultados se llegó a las siguientes conclusiones:

Para la variable habilidades sociales tenemos que el $96 \%$ de los participantes lo considera que se ubica en el nivel medio, esto debido a que los estudiantes se relacionan con todos desarrollando técnicas comunicativas que incluso les permite cooperar de forma amable y cortés, respetando las ideas de sus compañeros para la búsqueda de soluciones.

La dimensión comunicación afectiva en un $74 \%$ es considerado nivel medio, esto implica que se respeta las ideas entre compañeros, además de forma regular se dialoga y exponen sus ideas y sobre todo preguntan ante cualquier duda que se presente al momento de desarrollar las actividades.

Para la dimensión interacción social se evidencia que el $98 \%$ de los participantes considera que el nivel es medio, en tal sentido se evidencian actividades deportivas, sobre todo grupales, los estudiantes se comprometen a trabajar en equipo, respetan las normas de los juegos y sobre todo comparten gustos e intereses.

Para la dimensión desarrollo de sentimientos y emociones existe un $96 \%$ de los participantes que consideran en el nivel medio, es decir se respeta los sentimientos, preferencias e ideas de sus compañeros, además que permiten opinar sobre circunstancias indecisas sin llegar a la agresión.

Para la dimensión resolución de conflictos tenemos un 96\% de los participantes que consideran en el nivel medio, esto es producto de los acuerdos que se ponen de manifiesto 
para la búsqueda de soluciones, respetando la participación y opiniones de sus compañeros.

Para la dimensión relaciones interpersonales existe un 96\% de los participantes que consideran en el nivel medio, esto es a consecuencia de expresa sus ideas utilizando lenguaje sencillo en donde se comprende debido a la fluidez y sobre todo a la claridad al momento de participar, incluso las actividades que se realizan las realizan de forma amena y divertida utilizando los recursos que tienen a disposición.

\section{REFERENCIAS BIBLIOGRÁFICAS}

Abrams, D., Tianwen, P., Odriozolaa, K., Chenga, A., Bakera, A., Srikanth, J., Kochalkaa, C., Vinod, M. (2016). Neural circuits underlying mother's voice perception predict social communication abilities in children. https://www.pnas.org/content/113/22/6295

Araujo, P. (2017). Habilidades sociales y desempeño docente en los institutos de educación superior tecnológico de la selva central. (Tesis de doctoral, Universidad Nacional del centro del Perú. http://repositorio.uncp.edu.pe/bitstream/hendle/UNCP/4241/Araujo\%20Chuco.p df? sequence $=1 \&$ is Allowed $=y$

Barrera, M., Berkel, C., \& Castro, F. G. (2017). Directions for the advancement of culturally adapted preventive interventions: Local adaptations, engagement, and sustainability. Prevention 18(6), 640-648. http://dx.doi.org/10.1007/s11121-016-0705-9

Domínguez, C. (2015). La lúdica: una estrategia pedagógica depreciada. http://www3.uacj.mx/DGDCDC/SP/Documents/RTI/2015/ICSA/La\%20ludica.p $\mathrm{df}$

Escales, R., y Pujantell, M. (2016). Habilidades sociales. Madrid: Macmillan Iberia, S.A. García, A. y J. Llull (2009). El juego infantil y su metodología. Madrid: Ed. Editex.

García, J. y Lòpez, M. (2018). Espacio Europeo de Educación Superior: Competencias profesionales y empleabilidad. Revista Iberoamericana de Educación, 46(9-10). http://rieoei.org/2444.htm

García, T. (2021, febrero 10). Cómo reforzar las habilidades sociales de los niños. La tercera. https://www.latercera.com/paula/como-reforzar-las-habilidadessociales-de-los-ninos/ 
Gardner, H. (1983). Estructuras de la mente. Teorías de las inteligencias múltiples (10 ed.). Colombia: Fondo de cultura Económica.

Hernández, R. Zapata, N., Mendoza, C. (2013). Metodología de la investigación para bachillerato. Enfoque por competencias. México: McGraw-Hill/Interamericana Editores.

Hernández, R., Mendoza, C. (2018). Metodología de la investigación. Las rutas cuantitativa, cualitativa y mixta. México: McGraw-Hill/Interamericana Editores.

Hidalgo, C y Abarca, M. (1999). Comunicación Interpersonal: Programa de entretenimiento en habilidades sociales. Bogotá: ALFAOMEGA.

Lakatos, I. (1998). La metodología de los programas de investigación científica. Madrid: Alianza Universidad.

Latorre, A.; Delio del Rincón, I.; Arnal, J. (1996). Bases metodológicas de la investigación educativa. Barcelona: GR92.

Lederach, J. (2000). El abecé de la paz y los conflictos, Los libros de La Catarata.

Martínez, A. (2021, abril 14). Universidad de Especialidades Espíritu Santo realiza "Encuentro Internacional de Escuela Únicas. https://www.metroecuador.com.ec/ec/estilo-vida/2021/04/14/universidad-deespecialidades-espiritu-santo-realiza-encuentro-internacional-de-escuelaunicas.html

Martínez, M. (2010). El juego como método de aprendizaje. Revista digital Enfoques Educativos. Editorial Enfoques Educativos. http://www.enfoqueseducativos.es/enfoques/enfoques_71.pdf\#page=102

Olivos, X. (2017). Entrenamiento de Habilidades Sociales para la Integración Psicosocial de Inmigrantes. Tesis Doctoral. Madrid, España.

Rentería, J. (2018). Habilidades sociales para mejorar la convivencia escolar en docentes y estudiantes de la institución educativa "Santa Lucía" de Ferreñafe. (Tesis de maestría, Universidad César Vallejo). https://repositorio.ucv.edu.pe/bitstream/handle/20.500.12692/30090/Renteria_CJ .pdf? sequence $=1 \&$ isAllowed $=\mathrm{y}$

Rios, T. (2019). Estrategias lúdicas para el desarrollo psicomotor en niños de 3 años de una institución educativa inicial-Pisco-Ica. 
https://repositorio.ucv.edu.pe/bitstream/handle/20.500.12692/38349/rios_pt.pdf? sequen

Roca, E. (2014). Socialización de estudiantes con Dificultades de Aprendizaje. Valencia: ACDE.

Rojas, J. (2001). Programa de juegos lúdicos. Barcelona.

UNESCO (2020). Estrategias y recursos para el desarrollo de las habilidades socioemocionales en contexto de Covid-19 (Webinar). https://es.unesco.org/news/estrategias-y-recursos-desarrollo-habilidadessocioemocionales-contexto-covid-19-webinar

UNICEF (2021). Primera infancia. Impacto emocional en la pandemia. UNICEF Argentina.

https://www.unicef.org/argentina/media/10606/file/Primera\%20infancia.\%20Im pacto\%20emocional\%20en\%201a\%20pandemia\%20.pdf

UNICEF (2021a). Importancia del desarrollo de habilidades transferibles en América Latina el Caribe. https://www.unicef.org/lac/media/21536/file/Importancia_Desarrollo_Habilidad es_Transferibles_ALC_v.actualizada_marzo2021.pdf 